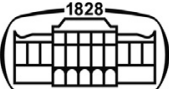

AKADÉMIAI KIADÓ

Acta Microbiologica et Immunologica Hungarica

67 (2020) 4, 201-208

DOl:

$10.1556 / 030.2020 .01200$

(C) 2020 The Author(s)

\section{RESEARCH ARTICLE}

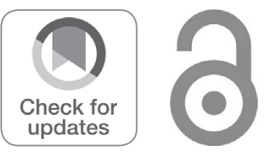

\title{
Distribution of enterovirus genotypes detected in clinical samples in Hungary, 2010-2018
}

\author{
ERIKA BUJAKI ${ }^{1}$, ÁGNES FARKAS ${ }^{1}$, ZITA RIGÓ $^{1}$ and \\ MÁRIA TAKÁCS ${ }^{1,2 *}$ (1) \\ ${ }^{1}$ Department of Virology, National Public Health Center, Budapest, Hungary \\ ${ }^{2}$ Institute of Medical Microbiology, Semmelweis University, Budapest, Hungary
}

Received: April 21, 2020 • Accepted: June 8, 2020

Published online: December 5, 2020

\begin{abstract}
This report provides the findings of a retrospective surveillance study on the emergence and circulation of enteroviruses with their associated clinical symptoms over a nine-year period detected at the National Enterovirus Reference Laboratory in Hungary between 2010-2018.

Enterovirus (EV) detection and genotyping were performed directly from clinical samples. From 4,080 clinical specimens $25 \mathrm{EV}$ types were identified with a median age of patients of 5 years and $68 \%$ of all cases affected children aged 10 years or younger, although infections occurred in all age-groups. In 130 cases neurological symptoms were recorded, in 123 cases the infection presented in skin related signs including hand, foot, and mouth disease (HFMD), herpangina and rash. In 2010 EV-A71 was found to cause the majority of diagnosed EV infections while in 2011 and from 2014-2018, Coxsackievirus (CV)-A6 was identified most often. Echovirus E6 accounted for the most cases in 2012 and Echovirus 30 dominated in 2013. EV-D68 was identified only in 2010 and 2013.

Widespread circulation of several EV-A and EV-B viruses with occasional occurrence of EV-C and EV-D was detected. The ability of EVs to cause severe infections in sporadic cases and regular outbreaks highlight the importance of continued monitoring of circulating EV types.
\end{abstract}

\section{KEYWORDS}

Enterovirus, direct detection, HFMD, neurological infection, Hungary

\section{INTRODUCTION}

The genus Enterovirus within the family Picornaviridae includes polioviruses (PV), coxsackieviruses (CV) A and B, echoviruses (E), and the numbered enteroviruses (EVs) adding up to more than 100 types. They are classified into four species, Enterovirus A to D, based largely on phylogenetic relationships in multiple genome regions [1].

EVs circulate worldwide causing sporadic or regular outbreaks of disease and can lead to significant morbidity and mortality, although most infections are subclinical. As $>90 \%$ of enterovirus infections are asymptomatic the infections with clinical symptoms represent less than $10 \%$ of enterovirus infections [2]. The individual genotypes are associated with different clinical manifestations and different enterovirus types have also been associated with the same clinical manifestations. Symptoms range from mild respiratory illness, skin rash, hand, foot, and mouth disease (HFMD), through myocarditis, meningitis and encephalitis, to severe neonatal sepsis-like disease and acute flaccid paralysis/myelitis (AFP/AFM), while the most common neurological manifestation is aseptic meningitis [3, 4].

HFMD, a disease characterized by vesicular exanthema on the hands, feet, and oral

mucosa was mostly linked to CV-A16 and EV-A71 occasionally leading to severe or fatal complications [5]. The widely circulating CV-A6 was previously generally linked to mild or asymptomatic infections, although it was known to cause herpangina (HA), an illness exhibiting painful mouth blisters. A change in the clinical phenotype of CV-A6 became evident when a large outbreak of HFMD caused by CV-A6 in Finland in 2008 was reported 
[6]. This was followed by several reports of CV-A6 associated HFMD from Europe [7-9], North America [10, 11] and Asia [12-15]. In the last decade CV-A10 was also reportedly an agent of HFMD [16, 17].

Species B EVs have been widely described as the most common cause of aseptic meningitis cases and outbreaks [3, $18,19]$ Clinical presentation of enterovirus meningitis varies with the patient's age and immune status [20]. As well as neurological infections, Enterovirus B viruses were also implicated in pneumonitis, pancreatitis, dilated myocarditis, and exanthems, including HFMD [3, 21, 22].

Severe respiratory infections linked to EV-D68, a species $\mathrm{D}$ enterovirus, have been described in Europe and North America in 2010, 2014 and 2016 [23, 24], including outbreaks associated with AFP/AFM (reviewed in [25]).

The classical method to diagnose Enterovirus (EV) infection has been virus isolation by cell culture from clinical specimens, followed by cross-neutralisation assay to determine the serotype [26]. The relationships between sequence data and enterovirus serotypes was first shown in the 1990s (reviewed in [27]) and was confirmed by several subsequent studies, hence enterovirus identification became synonymous with genome amplification and sequencing (reviewed in [28]).

Although EV surveillance is mainly carried out based on the recommendations set out by the World Health Organization (WHO) as a component of the Global Poliovirus Elimination Action Plan, it also provides parallel data on non-polio EV detection. In Europe non-polio EV surveillance commonly focuses on EV detected in specimens from hospitalized individuals with neurological infections [29].

The ability of EVs to cause large outbreaks, severe neurological infections and considerable disease burden highlight the need for the monitoring of EV types associated with severe or specific clinical presentations.

\section{MATERIALS AND METHODS}

\section{Samples}

The National Enterovirus Reference Laboratory receives specimens for diagnostic EV testing in cases of AFP/AFM symptoms, from hospitalized patients with severe neurological infections or otherwise suspected EV etiology i.e. HFMD, viral meningitis. Duplicate samples and repeat testing from the same patient were excluded from typing. Unfortunately, the specific clinical signs, acute or otherwise, did not always accompany the sample received.

\section{Detection and typing of enteroviruses}

Viral nucleic acids purified directly from the clinical specimens were first tested with pan-enterovirus nested RT-PCR targeting the $5^{\prime}$ untranslated region to detect enteroviral RNA following the protocol from Kapusinszky et al. [30]. Positive specimens were inoculated onto susceptible cell lines for poliovirus exclusion. EVs were directly typed by partial amplification and subsequent sequencing of the VP1 genome region using previously published primers [30-32] or, when necessary, by sequencing the diagnostic PCR amplicon. Viral sequences were subjected to nucleotidenucleotide BLAST $^{\circledR}$ analysis using the online server to determine genotype.

\section{Data collection}

All data accompanying the received specimen were recorded on arrival. Information about symptoms and clinical signs provided by the clinician requesting the test were categorised for this study with assistance from a medical professional based on the affected organ or leading symptom into the following categories: neurological, skin, HFMD, cardiac, sepsis, enteric, fever, exitus, unknown.

\section{RESULTS}

Between 2010 and 2018 an average of 453 clinical samples were received annually for EV testing, with the lowest number (340 tests) requested in 2012 and the most (656 tests) in 2018 (data not shown). Of these, an average of $9.5 \%$ were found to be EV-positive. The detection rate varied between $4.7 \%$ (19 positives of 405 samples) in 2015 and $17.6 \%$ ( 85 positives of 482 ) in 2016.

During the 9 years study period, 387 clinical specimens out of the 4,080 received samples tested positive for EV, corresponding to 284 cases. Typing was unsuccessful in 6 cases and in three cases EV type could only be determined to genogroup level ( 1 of species A, 2 of species B). In $1 \mathrm{CV}-\mathrm{B}$ case and in 3 echovirus cases the actual genotype could not be identified. 25 different EVs were identified, with the majority, 14 types belonging to species $\mathrm{B}$, followed by 8 types of species A, two types of species $C$ and one type of species D. Between 2010 and 2018 the most frequently detected genotype in Hungary was CV-A6, identified in $40.1 \%$ of all cases, the second most frequent type was E-30, with $8.5 \%$, the third, EV-A71 representing $7.8 \%$ of all cases, followed by CV-A16 detected in $7.4 \%$ of cases (Table 1 ).

The most frequently detected species and EV types varied between the years during the study period. In 2012 and 2013 species B were identified more often than other species, whilst in all the other seven years more cases were linked to Genogroup A than to Genogroup B. In 2010 EVA71 caused most reported infections, in 2011 and 20142018 the majority of EV infections were linked to CV-A6. Echovirus 6 and 30 were detected in most enteroviral infection cases in 2012 and 2013 causing 25 and $42.4 \%$ of yearly reported infections respectively. CV-A16 was the second most frequent EV type identified in 5 years (201012, 2016-17) while EV-A71 was among the top three EV type 3 times with both CV-A16 and EV-A71 being detected in 7 of the 9 years of the study. Coxsackievirus B genotypes represented $7.4 \%$ of all $\mathrm{EV}$ positive cases, causing the most reported infections in 2012, while in 2011 and 2015 there was no CV-B detected. Eight different Echovirus types were found with 6 of them occurring in more than one year. E-6 
Table 1. Case numbers of detected enterovirus (EV) types in Hungary, 2010-2018

\begin{tabular}{|c|c|c|c|c|c|c|c|c|c|c|c|}
\hline & Type & 2010 & 2011 & 2012 & 2013 & 2014 & 2015 & 2016 & 2017 & 2018 & 2010-2018 \\
\hline \multirow[t]{9}{*}{ Species A } & EV-A71 & 10 & 3 & & 3 & 1 & & 2 & 2 & 1 & 22 \\
\hline & CV-A2 & & & & 1 & & & & 1 & & 2 \\
\hline & CV-A4 & & & & & 1 & & 3 & 1 & 1 & 6 \\
\hline & CV-A5 & & & & & 2 & 1 & & & & 3 \\
\hline & CV-A6 & 1 & 8 & & 4 & 16 & 6 & 38 & 22 & 19 & 114 \\
\hline & CV-A8 & & & & & & & & & 1 & 1 \\
\hline & CV-A10 & & & & 1 & 5 & & 1 & 1 & & 8 \\
\hline & CV-A16 & 4 & 4 & 3 & 1 & & 1 & 4 & 4 & & 21 \\
\hline & $C V-A^{a}$ & & & & & & & & & 1 & 1 \\
\hline \multirow[t]{17}{*}{ Species B } & CV-B1 & & & 1 & & & & & & & 1 \\
\hline & CV-B2 & & & 3 & 2 & & & & & & 5 \\
\hline & CV-B3 & & & & & 2 & & & & & 2 \\
\hline & CV-B4 & & & 1 & & & & 2 & 1 & & 4 \\
\hline & CV-B5 & 1 & & 3 & & & & 1 & 1 & 2 & 8 \\
\hline & $C V-B^{a}$ & & & & 1 & & & & & & 1 \\
\hline & CV-A9 & & & & 1 & 1 & & 2 & & & 4 \\
\hline & E-3 & 1 & & & & & & & & & 1 \\
\hline & E-6 & 2 & 1 & 4 & & & 1 & 2 & & & 10 \\
\hline & E-9 & & & & 1 & & & & & 9 & 10 \\
\hline & E-11 & & & & 2 & 1 & 4 & & & 6 & 13 \\
\hline & E-18 & & & & & & & 1 & & & 1 \\
\hline & E-20 & & & & & & 1 & 1 & & & 2 \\
\hline & E-25 & 3 & & & & & & & & & 3 \\
\hline & E-30 & 2 & & & 14 & 2 & & 2 & & 4 & 24 \\
\hline & Echovirus $^{\mathrm{a}}$ & & & & & & & 2 & & 1 & 3 \\
\hline & EV-B ${ }^{\mathrm{a}}$ & & & 1 & & & 1 & & & & 2 \\
\hline \multirow[t]{2}{*}{ Species C } & CV-A13 & & & & & 1 & & & & & 1 \\
\hline & CV-A24 & & & & & & & 1 & & & 1 \\
\hline Species D & EV-D68 & 2 & & & 2 & & & & & & 4 \\
\hline Untyped $^{\mathrm{b}}$ & & & 1 & & & & & & 2 & 3 & 6 \\
\hline Total & All & 26 & 17 & 16 & 33 & 32 & 15 & 62 & 35 & 48 & 284 \\
\hline
\end{tabular}

Note:

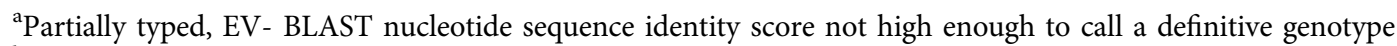

${ }^{\mathrm{b}}$ Diagnostic PCR positive, molecular typing unsuccessful

and E-30 were each detected in 5 of the nine years studied, causing most cases in 2012 and 2013 respectively. In 2012, the yearly sample number was well below average compared to the whole period. Each year either Genogroup A or B dominated the landscape with species C and D only sporadically detected. In 2016 five different echovirus types were identified in the received specimens.

Most EV-positive samples were received in the second half of the year (Fig. 1a). Overall numbers peaked in August and September (58 cases, $20.4 \%$ and 40 cases, $14.1 \%$ respectively) and lowest numbers of positive samples were observed between February and April (5-7 cases per month). There were differences in the monthly distribution of positive samples between the years. In 2016 most cases were recorded in November (20.1\%), while in 2017 more infections were detected during the last two months of the year, than during August and September. EV-D68 was detected in August and September (1 and 3 cases respectively). CV-A6 was detected throughout the year, while most cases occurred in September and December. EV detection, excluding CV-A6 cases, peaked in August and over a third of the reported infections occurred between September and
November. During the study period Echoviruses were detected in all months apart from March and April.

The age of patients with confirmed EV infection varied between 0 and 74 years, with a median age of 5 years. Most patients were aged between 1 and 5 years $(43 \%, n=122)$, followed by the age group of 6-15 years with $20.1 \%(n=$ $57)$, patients aged $26-45$ years $(15.1 \%, n=43)$ and young adults $16-25$ years $(10.2 \%, n=29)$. Children under 1 year represented $9.5 \%$ of all cases $(n=27)$. The least affected were adults over 45 years with $2.1 \%(n=6)$. Overall $52.5 \%$ of cases (149/284 EV positive cases) involved children of 5 years or younger. The 16 cases affecting babies under 3 months old included 3 cases each of CV-A16 and CV-B5 infections with the remaining 10 cases evenly distributed between 9 further types (CV-A4, CV-B1, CV-B3, CV-B4, CV-A9, E-3, E-11, E-18), leaving one such case typed as echovirus and one, that was untypeable by our methods. There was one case during the study period involving a person over 65 years, a 74-year-old patient with a CV-A4 infection. CV-A6 affected all age groups between 3 months and 65 years with $44.9 \%$ of all EV cases involving patients of 16 years or older linked to CV-A6. Echoviruses were 
a

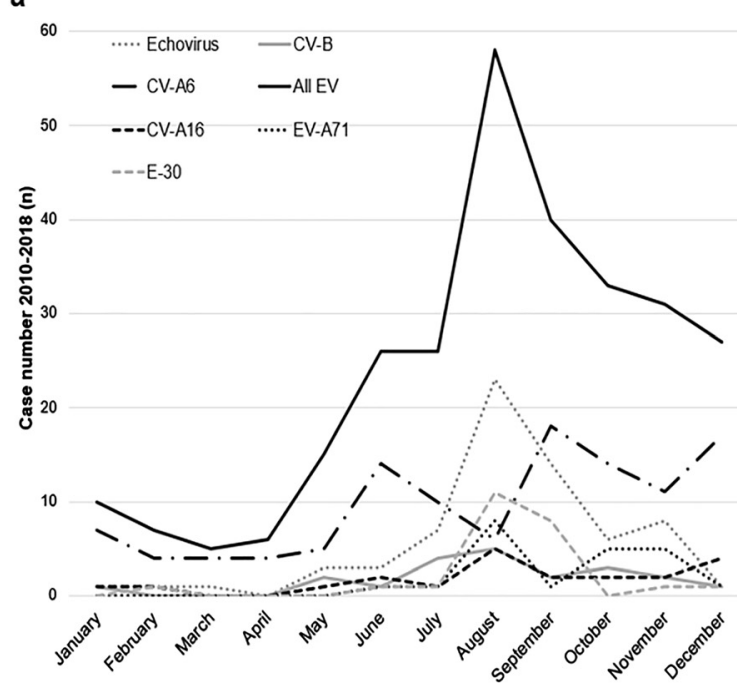

b

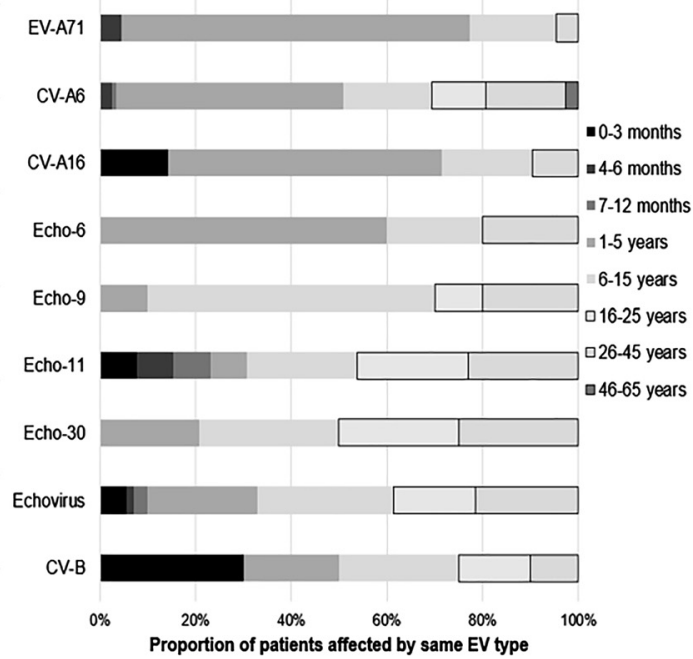

Fig. 1. Distribution of confirmed EV infections by "a" month of detection "b" age of patient

detected in samples from people of all age groups under 46 years with more cases affecting patients over 16 years than children of 5 years or younger (38.6 and $32.9 \%$ of reported echovirus cases respectively) (Fig. 1b). Two patients with confirmed EVD68 infections were aged 8 months, 1 patient was 6 years old and the fourth had an age of 9 years.

The most frequent specimen types that tested positive for EV were faeces/rectal swabs $(213,55 \%$ of 387 positive samples) and respiratory samples (102, 26.4\%), followed by cerebrospinal fluid $(61,15.8 \%)$, vesicle/skin swab (9, 2.3\%) and biopsy (2, 0.5\%) (Fig. 2). Most CV-A6 infections were confirmed in faeces $(63.2 \%, 96$ of $152 \mathrm{CV}-\mathrm{A} 6$ positive samples), followed by respiratory specimens (27\%, 41 positive samples), skin/vesicle swab (5.9\%, 9 samples) and CSF (3.9\%, 6 samples). The second highest number of EV positive samples, 39 specimens were received from patients infected with E-30. A third of EV-30 positive specimens were CFS samples (13 of 39 samples), slightly more, $41 \%$ were faecal samples (16 of 39 samples) and $25.6 \%$ (10 of 39 samples) were respiratory specimens. Very similar ratios were observed in the case of all detected echoviruses combined: $44.4 \%$ faecal samples, $34.3 \%$ CSF, $21.2 \%$ respiratory specimens (44, 34 and 21 echovirus positive samples respectively of a total of 99). The third and fourth most EV positive samples were taken from patients with EV-A71 and CV-A16 infections (30 and 27 samples). Over half of EVA71 and CV-A16 positive samples were faecal samples (19 of 30 samples 15 of 27 samples respectively), followed by 10 positive respiratory sample of each EV type, plus 1 CSF sample positive for EV-A71 and 2 CSF samples positive for CV-A16. Most CV-B infections were detected in faecal samples (14 samples, $45.2 \%$ ), followed by CSF (8 samples, $25.8 \%$ ), respiratory specimens (7 samples, 22.6\%) and postmortem tissue samples (2 specimens, 6.5\%). EV-D68 was identified in 4 cases of EV infection, corresponding to 2 faecal, 2 CSF and 2 respiratory specimens.

Neurological symptoms were recorded as affecting the individuals with detected EV infection most often (recorded
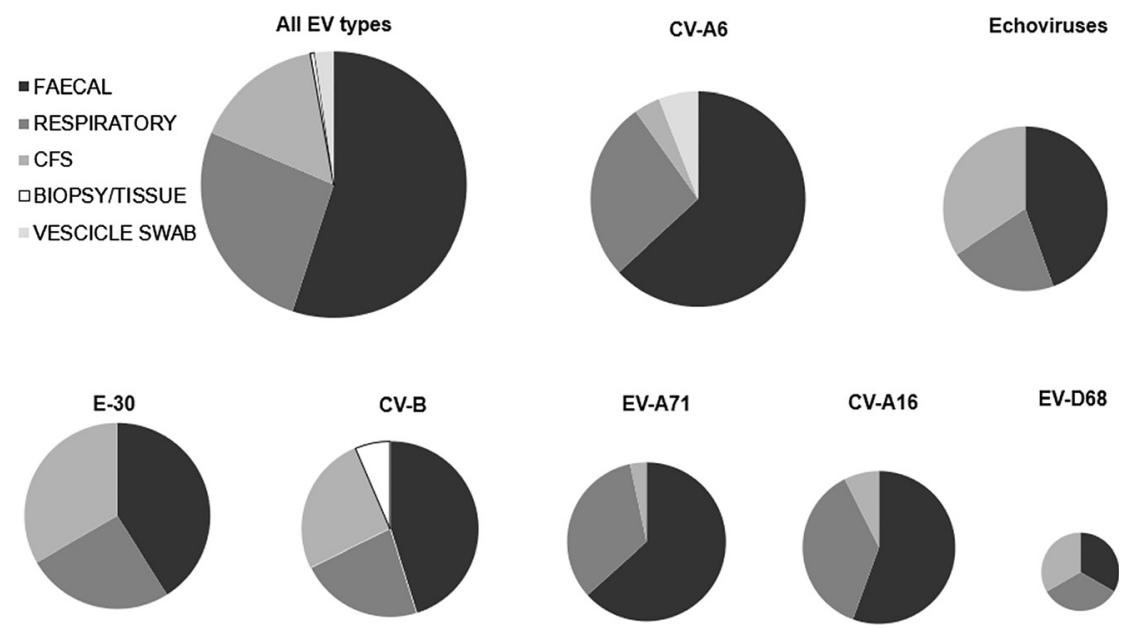

EV-D68

Fig. 2. Specimen types from infections with common EV types 
in 130 cases), followed by symptoms affecting the skin or mucous membrane (123 cases), including cases of HFMD (55 cases) (Fig. 3). Species B EVs were detected in most cases with neurological symptoms $(60 \%, 78$ cases of 130$)$, while over a third were linked to Species A viruses (35.4\%, 46/ 130). EV-D68 infections were proved in 3 cases in patients with neurological symptoms and in 1 case with non-specific fever. Both detected infections by Species C EVs, one case of $\mathrm{CV}-\mathrm{A} 13$ and one of $\mathrm{CV}-\mathrm{A} 24$ infection were received from individuals suffering from neurological symptoms. In 13 cases of detected EV infections information regarding symptoms were not available to the laboratory. Skin related symptoms were affecting individuals with infection of one of 9 EV types (CV-A6, CV-A10, CV-A16, CV-A4, EV-A71, CV-A2, CV-B4, E-9, E-11), while HFMD were only related to three EV types, CV-A6, CV-A10 and EV-A71. Cardiac problems were reported in 9 cases and a third of these, 3 cases were linked to E-11 infections, in the rest of the samples received from these individuals EV-A71, CV-A6, CV-A5, CV-B4 and E-9 were identified. Enteric symptoms accompanied 6 cases of EV infections, with Enterovirus A identified in all of them. The laboratory detected EV in specimens from two cases of sepsis, one was linked to E-3, the other to E-11 infection. CV-B5 was found in samples received from the one recorded fatal case. Non-specific fever was the lone recorded symptom in four patients with EV positive samples. One patient with EV-A71 infection suffered neurological and cardiac symptoms simultaneously, while another individual with EV-A71 infection had a combination of enteric and neurological symptoms. Exanthems and neurological symptoms were both recorded in one case of CV-A6 and in one case of CV-B4 infection.

\section{DISCUSSION}

This study reports EV types detected in clinical specimens in Hungary during a nine-year period. It also provides information in association with patients' age and symptoms. Results demonstrate the circulation of several EV-A and EV$\mathrm{B}$ viruses, with occasional occurrence of EV-C and EV-D

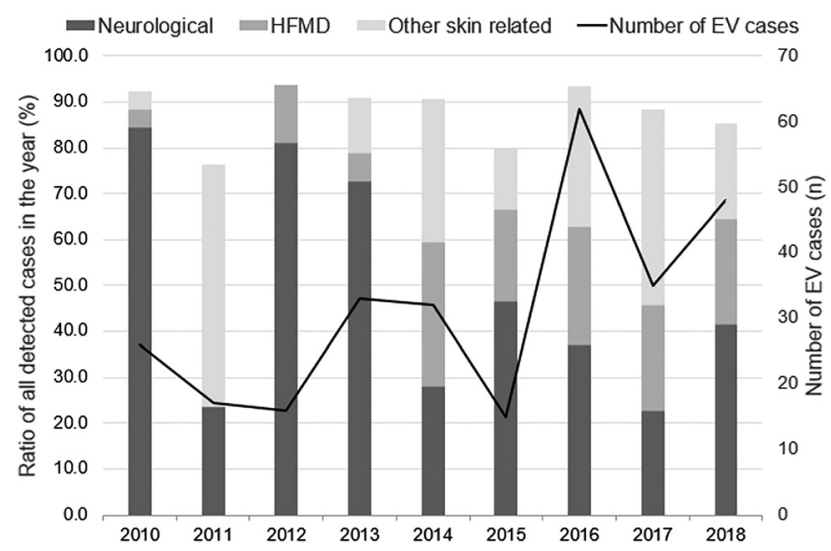

Fig. 3. Leading symptoms associated with detected EV cases strains. During the study period frequent changes in EV type frequencies were observed.

Reports on national enterovirus detection data from European countries from the past decade is scarcely available. Most studies focus either on certain symptoms (aseptic meningitis or HFMD) or EV type (CV-A6, EV-A71 or EVD68 mostly), often reporting regional as opposed to country-wide results. Our data represents the results of testing specimens received from across the whole country but is not a result of a national EV surveillance programme. Current legislation in Hungary orders samples to be sent to the national reference laboratory for investigation primarily in cases of neurological symptoms indicative of AFP.

The 25 EV types detected in this study differed slightly from those of a previous study of enteroviruses in Hungary, when 29 samples from 28 patients diagnosed with hand, foot and mouth disease, meningitis and encephalitis were molecularly typed. As a result between June 2000 and August 2008 echovirus type 2, 4, 6, 7, 9, 11, 13, 25, 30, CVA16, -A19, -B5, and EV-A71 were reported [30]. Differences between the variety of EV types identified could be explained by the great difference in sample numbers and the 10 years shift between the study periods. Detected EV genotypes in the 2000-2008 Hungarian study showed great alignment with results of a surveillance report from the Netherlands between 1996 and 2011 enteroviruses of species B were the major cause of enterovirus infections reported and for several types sharp increases in the number of reports occurred at intervals of $\sim 2-3$ years [33]. In Hungary in 2010 and 2011 more infections with EV-A than with EV-B were detected, although lower than average case numbers were observed in those years with 26 and 17 yearly cases respectively, while in the Netherlands species $B$ viruses dominated in both years.

A study by the European Centre for Disease Control collected data from 24 European countries on non-polio EVs detected during a 3-year study period revealed 66 types of EVs of which 16 types were also detected in Hungary [34]. The most frequently reported enterovirus type between 2015-2017 in both studies was CVA6, representing 13\% of typed EVs in Europe and 59\% of detected EV infections in Hungary. These results highlight the prevalence and widespread circulation of CVA6.

In Spain 34 different EV types were identified (26 from EV species B and 8 from species A) during a 4-year study period (2010-2013) in $1430 \mathrm{EV}$ positive samples [35]. In contrast, during the same period in $\sim$ Hungary 18 EV types were detected (11 types of EV-B, 5 types of EV-A and 1 type of EV-D) in 92 reported EV infection cases with $134 \mathrm{EV}$ positive clinical samples.

EV infections occurred in all age groups of patients and the group most affected was 1-5 years, younger than in earlier reports from France [36], but older than previously found in USA [37]. In the current study the proportion of infections affecting neonates under 3 months at 5.6\% was lower than in Spain (17\%) [35] or the Netherlands (46.8\%) [38], which might indicate a lower detection rate in this age group. 
According to our data, since 2014 samples from enterovirus infections from patients with symptoms affecting skin on average accounted for $62 \%$ of all yearly cases, apart from year 2015, when it was $33.3 \%$, but total received sample number fell well below study average. During the past decade CVA6 has become the predominant pathogen causing HFMD across Europe and Asia. We detected CV-A6 in all study years, apart from 2012, but the first confirmed Hungarian HFMD associated with CV-A6 was only received in 2013, 5 years after the CV-A linked HFMD in Finland [6]. During the first four years of our study all, but one CV-A6 was detected in patients with skin related symptoms including hand-foot-and-mouth disease (HFMD), while after 2015 solely neurological symptoms were recorded in 5-22\% of individuals with CV-A6 infections per year. Atypical HFMD was linked to newly emerging variants of CV-A6 infection in children in 2014 [39]. Novel recombinant forms of the virus have been linked to clinically unusual HFMD and are believed to be circulating widely and contributing to an increased incidence of HFMD worldwide [40]. CA6-associated HFMD is also characterized by a high incidence in adults, with onset in winter and a tendency to experience onychomadesis (shedding of the nails) after recovery [41]. In our study in $43 \%$ of cases of CV-A6 infections HFMD was reported, while a more general, skin or mucous membrane related symptom was noted in $36 \%$ of CV-A6 cases. The yearly ratio between the two symptom categories varied greatly. Without the availability of detailed and accurate description of all symptoms it cannot be determined which, if any cases could be attributed in presentation to atypical HFMD as outlined before [11] or herpangina. There are no surveillance data available on the incidence of HFMD in Hungary since it is not a notifiable disease.

Co-circulation of CV-A6 with CV-A10 and CV-A16 was already reported previously $[7,39]$ and in a study of HFMD cases in Poland between 2013-2016 the emergence and cocirculation of CV-A6 with CV-A10 and CV-A16 was also confirmed [9]. In our study CV-A6, CV-A16 and EV-71 were the only three types identified in specimens associated with HFMD, however only CV-A6 was detected in any HFMD samples since 2013. CV-A10 was also detected in 6 cases with mouth blisters and exanthems affecting the torso or the palms and soles.

Among the EV-B viruses detected in Hungary during the study period E-30, E-11, E-9 and E-6 dominated, these 4 types were also in the overall top 7 most often identified EVs. This data shows a good correlation to those reported elsewhere, even if they cover different periods. In an Amsterdam hospital (2007-2011) five echoviruses E-30, E25, E-6, E-9 and E-7 ranked among the top 10 types found overall [38]. Echoviruses were also among the types detected most frequently as part of hospital-based enterovirus surveillances in France (2000-2004) and the USA (1970-2005) $[33,34]$. E-30, E-9, and E- 6 followed the predominant E-4 in a study that investigated the etiology of aseptic meningitis and infant febrile syndromes in 2008 in Spain, [42]. Similarly, in the Slovak Republic a program monitoring sewage water and AFP cases between 2001-2011 revealed the dominance of CV-Bs (40\%) and echoviruses (37\%, mostly E3, E-11 and E-7) [43].

Between 2011 and 2014 in samples from enteroviral aseptic meningitis from two regions in Poland the three predominant EV types identified by serological methods were E-30, E-6, and E-11, with E-7, CV-A9 and CVB viruses also detected [44]. Results during the same period in Hungary were similar with slight variation in EV types. In Hungary EV-A71 was identified in most reported EV infections from patients with neurological symptoms in 2011, while EV-B viruses (E-6, E-30, CV-B3) dominated between 2012-14, with E-9 and E-11 also detected.

In another nine-year study in Austria (1999-2007) E-30 accounted for most cases (26.5\%) of AFP or aseptic meningitis, with fifteen more echovirus serotypes also detected. Cases of infection with CV-B strains accounted for $18.8 \%$ and CV-A viruses for $18.2 \%$, while EV71 was found in $8.8 \%$ [45]. In the current study in Hungary too, E-30 was detected in most cases where neurological symptoms were recorded, however EV-71 and CV-A viruses accounted for slightly more, $14.6 \%$ and $22.3 \%$ respectively, while CV-B strains were detected in less, $13.1 \%$ of such cases.

For some echovirus serotypes $(6,9,11$, and 30$)$, as well as for CV-B4 and 5, periodicity in detection or increase in case numbers with intervals of a few years could be observed, although case numbers were not always high enough to positively establish circulation patterns for comparison with earlier experiences reported.

The higher than average annual case numbers due to the increase in the detection rate of E-30 in 2013 and to CV-A6 in 2014 and 2016-2018 could be attributed to outbreaks and endemic circulation respectively.

In our study EV-D68, which primarily causes respiratory infections, was identified only in 2010 and 2013, in years, when samples from patients with neurological symptoms still counted for over two-thirds of total processed samples. EV-D68 cases might have been missed because specimens from patients with respiratory diseases are usually not tested for $\mathrm{EV}$ infection. As it was established, existing diagnostic screening of stool samples together with occasional CSF samples was ineffective for the detection of EV-D68 as this EV type is only rarely detected in stool or CSF samples [46], which can also explain the low detection rate experienced during the study period.

\section{CONFLICT OF INTEREST}

The authors declare that they have no conflict of interest.

\section{AUTHORS CONTRIBUTIONS}

Erika Bujaki and Mária Takács contributed to the study conception and design. Material preparation, data collection and analysis were performed by Erika Bujaki, Ágnes Farkas and Zita Rigó. The first draft of the manuscript was written 
by Erika Bujaki and all authors commented on previous versions of the manuscript. All authors read and approved the final manuscript.

\section{ACKNOWLEDGEMENT}

The authors would like to thank Ágota Wellerné Pus and Anna Marchut for their practical work in enteroviral diagnostic testing.

\section{REFERENCES}

[1] Knowles N, Hovi T, Hyypiä T, King A, Lindberg A, Pallansch M. Picornaviridae. In: King AMQ, Carstens AMJ, Lefkowits EB, editors Virus taxonomy: classification and nomenclature of viruses, ninth report of the international committee on taxonomy of viruses. San Diego: Elsevier; 2012. pp. 855-80.

[2] Nathanson N, Kew OM. From emergence to eradication: the epidemiology of poliomyelitis deconstructed. Am J Epidemiol 2010; 172: 1213-29. https://doi.org/10.1093/aje/kwq320.

[3] Pallansch M, Oberste M, Whitton J. Enteroviruses: polioviruses, coxsackieviruses, echoviruses, and newer enteroviruses. In: Knipe D, Howley P, editors Fields virology 2, 6th ed. Lippincott Williams \& Wilkins; 2013. pp. 490-530.

[4] Tapparel C, Siegrist F, Petty TJ, Kaiser L. Picornavirus and enterovirus diversity with associated human diseases. Infect Genet Evol 2013; 14: 282-93. https://doi.org/10.1016/j.meegid.2012.10.016.

[5] Solomon T, Lewthwaite P, Perera D, Cardosa MJ, Mcminn P, Ooi $\mathrm{MH}$, et al. Virology, epidemiology, pathogenesis, and control of enterovirus 71. Lancet Infect Dis 2010; 10: 778-90. https://doi.org/ 10.1016/S1473-3099(10)70194-8.

[6] Österback R, Vuorinen T, Linna M, Susi P, Hyypiä T, Waris M. Coxsackievirus A6 and hand, foot, and mouth disease, Finland. Emerg Infect Dis 2009; 15: 1485-8. https://doi.org/10.3201/ eid1509.090438.

[7] Mirand A, Henquell C, Archimbaud C, Ughetto S, Antona D, Bailly JL, et al. Outbreak of hand, foot and mouth disease/herpangina associated with coxsackievirus A6 and A10 infections in 2010, France: a large citywide, prospective observational study. Clin Microbiol Infect 2012; 18:E110-18. https://doi.org/10.1111/j. 1469-0691.2012.03789.x.

[8] Puenpa J, Vongpunsawad S, Waris M, Eriksson E, Albert J, Midgley S, et al. Molecular epidemiology and the evolution of human coxsackievirus A6. J Gen Virol 2016: 3225-31. https://doi. org/10.1099/jgv.0.000619.

[9] Wieczorek M, Ciạćka A, Krzysztoszek A, Figas A, Szenborn L. Genetic characterization of human enteroviruses associated with hand, foot and mouth diseases in Poland, 2013 - 2016. Pol J Microbiol 2017; 66: 405-9. https://doi.org/10.5604/01.3001.0010. 4889.

[10] McIntyre M, Stevens K, Davidson S. Notes from the field: severe hand, foot, and mouth disease associated with coxsackievirus A6 Alabama, Connecticut, California, and Nevada, November 2011February 2012. MMWR Morb Mortal Wkly Rep 2012; 61: 213-4. https://doi.org/10.1099/jgv.0.000619.
[11] Lott JP, Liu K, Landry M, Nix WA, Oberste MS, Bolognia J, et al. Atypical hand-foot-mouth disease associated with coxsackievirus A6 infection. J Am Acad Dermatol 2013; 69: 736-41. https://doi. org/10.1016/j.jaad.2013.07.024.

[12] Tsuguto F, Iizuka S, Enomoto M, Abe K, Yamashita K, Hanaoka $\mathrm{N}$, et al. Hand, foot, and mouth disease caused by Cox- sackievirus A6. Emerg Infect Dis 2012; 18: 10-2. https://dx.doi.org/10.3201/ eid1802.111147.

[13] Hober D, Sauter P. Pathogenesis of type 1 diabetes mellitus: interplay between enterovirus and host. Nat Rev Endocrinol 2010; 6: 279-89. http://www.nature.com/doifinder/10.1038/nrendo.2010.27.

[14] Li Y, Chang Z, Wu P, Liao Q, Liu F, Zheng Y, et al. Emerging enteroviruses causing hand, foot and mouth disease, China, 20102016. Emerg Infect Dis 2018; 24(1-5). https://doi.org/10.3201/ eid2410.171953.

[15] Ji T, Han T, Tan X, Zhu S, Yan D, Yang Q, et al. Surveillance, epidemiology, and pathogen spectrum of hand, foot, and mouth disease in mainland of China from 2008 to 2017, Biosaf Heal 2019; 1(1): 32-40, https://doi.org/10.1016/j.bsheal.2019.02.005.

[16] Blomqvist S, Klemola P, Kaijalainen S, Paananen A, Simonen M-L, Vuorinen T, et al. Co-circulation of coxsackieviruses A6 and A10 in hand, foot and mouth disease outbreak in Finland. J Clin Virol 2010; 48: 49-54. https://doi.org/10.1016/j.jcv.2010.02.002.

[17] Aswathyraj S, Arunkumar G, Alidjinou EK, Hober D. Hand, foot and mouth disease (HFMD): emerging epidemiology and the need for a vaccine strategy. Med Microbiol Immunol 2016; 205 : 397-407. https://doi.org/10.1007/s00430-016-0465-y.

[18] Holmes CW, Koo SSF, Osman H, Wilson S, Xerry J, Gallimore CI, et al. Predominance of enterovirus B and echovirus 30 as cause of viral meningitis in a UK population. J Clin Virol 2016; 81: 90-3. https://doi.org/10.1016/j.jcv.2016.06.007.

[19] Nougairede A, Bessaud M, Thiberville S, Piorkowski G, Ninove L, Zandotti C, et al. Widespread circulation of a new echovirus 30 variant causing aseptic meningitis and non-specific viral illness, South-East France, 2013. J Clin Virol 2014; 61(1): 118-24. https:// doi.org/10.1016/j.jcv.2014.05.022.

[20] Modlin J. Coxsackieviruses, echoviruses, and newer enteroviruses. In: Mandell G., Bennett J., Dolin E., editors Mandell, Douglas, and Bennett's principles and practice of infectious diseases, 4th ed. New York: Churchill Livingstone; 2000. pp. 1904-19.

[21] Abzug MJ. The enteroviruses: problems in need of treatments. J Infect 2013; 68: 108-14. https://doi.org/10.1016/j.jinf.2013.09.020.

[22] Lei X, Cui S, Zhao Z, Wang J. Etiology, pathogenesis, antivirals and vaccines of hand, foot, and mouth disease. Natl Sci Rev 2015; 2. https://doi.org/10.1093/nsr/nwv038.

[23] Piralla A, Girello A, Grignani M, Gozalo-margu M. Phylogenetic characterization of enterovirus 68 strains in patients with respiratory syndromes in Italy. J Med Virol 2014; 1593: 1590-3. https:// doi.org/10.1002/jmv.23821.

[24] Greninger AL, Naccache SN, Messacar K, Clayton A, Yu G, Somasekar S, et al. A novel outbreak enterovirus D68 strain associated with acute flaccid myelitis cases in the USA (2012-14): a retrospective cohort study. Lancet Infect Dis 2015; 15: 671-82. https://doi.org/10.1016/S1473-3099(15)70093-9.

[25] Holm-Hansen CC, Midgley SE, Fischer TK. Review Global emergence of enterovirus D68: a systematic review. Lancet Infect Dis 2016; 16: 64-75. https://doi.org/10.1016/S1473-3099(15) 00543-5 Virology. 
[26] Rotbart H, Romero J. Laboratory diagnosis of enteroviral infections. In: Robart $\mathrm{H}$, editor Human enterovirus infections. Washington: ASM Press; 1995. pp. 401-18.

[27] Hyypiä T, Hovi T, Knowles NJ, Stanway G. Classification of enteroviruses based on molecular and biological properties. J Gen Virol 1997; 78: 1-11. https://doi.org/10.1099/0022-1317-78-1-1.

[28] Harvala H, Broberg E, Benschop K, Berginc N, Ladhani S, Susi P, et al. Recommendations for enterovirus diagnostics and characterisation within and beyond Europe. J Clin Virol 2018; 101: 11-7. https://doi.org/10.1016/j.jcv.2018.01.008.

[29] Harvala H, Jasir A, Penttinen P, Celentano LP, Greco D, Broberg E. Surveillance and laboratory detection for non-polio enteroviruses in the European Union/European Economic Area, 2016. Euro Surveill 2017; 22(45): 1-13. https://doi.org/10.2807/15607917.ES.2017.22.45.16-00807.

[30] Kapusinszky B, Szomor KN, Farkas A, Takacs M, Berencsi G. Detection of non-polio enteroviruses in Hungary 2000-2008 and molecular epidemiology of enterovirus 71, coxsackievirus A16, and echovirus 30. Virus Gene 2010; 40: 163-73. https://doi.org/10. 1007/s11262-009-0440-4.

[31] Allan Nix W, Oberste MS, Pallansch MA. Sensitive, seminested PCR amplification of VP1 sequences for direct identification of all enterovirus serotypes from original clinical specimens. J Clin Microbiol 2006; 44: 2698-704. https://doi.org/10.1128/JCM. 00542-06.

[32] Bessaud M, Jegouic S, Joffret ML, Barge C, Balanant J, Gouandjika-Vasilache I, et al. Characterization of the genome of human enteroviruses: design of generic primers for amplification and sequencing of different regions of the viral genome. J Virol Methods 2008; 149: 277-84. https://doi.org/10.1016/j.jviromet. 2008.01.027.

[33] Van Der Sanden SMG, Koopmans MPG, Van Der Avoort HGAM. Detection of human enteroviruses and parechoviruses as part of the national enterovirus surveillance in the Netherlands, 19962011. Eur J Clin Microbiol Infect Dis 2013: 1525-31. https://doi. org/10.1007/s10096-013-1906-9.

[34] Bubba L, Broberg EK, Jasir A, Simmonds P, Harvala H, Redlberger-Fritz M, et al. Circulation of non-polio enteroviruses in 24 EU and EEA countries between 2015 and 2017: a retrospective surveillance study. Lancet Infect Dis 2019; 3099. https://doi.org/ 10.1016/S1473-3099(19)30566-3.

[35] Cabrerizo M, Díaz-Cerio M, Munoz-Almaro C, Rabella N, Tarragó D, Pilár Romero M, et al. Molecular epidemiology of enterovirus and parechovirus infections according to patient age over a 4-year period in Spain. J Gen Virol 2017; 89: 435-42. https://doi.org/10.1002/jmv.24658.

[36] Antona D, Leveque N, Chomel JJ, Dubrou S, Levy-Bruhl D, Lina B. Surveillance of enteroviruses in France, 2000 - 2004. Eur J Clin
Microbiol Infect Dis 2007: 403-12. https://doi.org/10.1007/ s10096-007-0306-4.

[37] Khetsuriani N, Lamonte-Fowlkes A, Oberst S, Pallansch MA. Enterovirus surveillance-United States, 1970-2005. MMWR Surveill Summ 2006; 55: 1-20. https://www.cdc.gov/mmwr/preview/ mmwrhtml/ss5508a1.htm.

[38] Janes VA, Minnaar A, Koen G, van Eijk H, Dijkman-de Haan K, Pajkrt D, et al. Presence of human non-polio enterovirus and parechovirus genotypes in an Amsterdam hospital in 2007 to 2011 compared to national and international published surveillance data: a comprehensive review. Euro Surveill 2014: 1-9. https://doi. org/10.2807/1560-7917.ES2014.19.46.20964.

[39] Sinclair C, Gaunt E, Simmonds P, Broomfield D, Nwafor N, Wellingotn L, et al. Atypical hand, foot, and mouth disease associated with coxsackievirus A6 infection, Edinburgh, United Kingdom, January to February 2014. Euro Surveill 2014; 19: 20745. https://doi.org/10.2807/1560-7917.ES2014.19.12.20745.

[40] Gaunt E, Harvala H, Riikka O, Vattipally BS, Thomson E, Waris $\mathrm{M}$, et al. Genetic characterization of human coxsackievirus A6 variants associated with atypical hand, foot and mouth disease: a potential role of recombination in emergence and pathogenicity. J Clin Virol 2015; 96: 1067-79. https://doi.org/10.1099/vir.0.000062.

[41] Bian L, Wang Y, Yao X, Mao Q, Xu M, Liang Z. Coxsackievirus A6: a new emerging pathogen causing hand, foot and mouth disease outbreaks worldwide. Expert Rev Anti Infect Ther 2015; 13: 1061-71. https://doi.org/10.1586/14787210.2015.1058156.

[42] Cabrerizo M, Trallero G, Echevarría J, Moreno-Docón A, Pena M, Pérez-Ruiz $M$, et al. Molecular characterization of enteroviruses associated with neurological infections in Spain, 2008. J Med Virol 2013; 85: 1975-7. https://doi.org/10.1097/INF.0b013e31828bbe46.

[43] Klement C, Kissova R, Lengyelova V, Stipalova D, Sobotova Z, Galama JMD, et al. SHORT REPORT Human enterovirus surveillance in the Slovak Republic from 2001 to 2011. Epidemiol Infect 2013; 141: 2658-62. https://doi.org/10.1017/ S0950268813000563.

[44] Wieczorek M, Figas A, Krzysztoszek A. Enteroviruses associated with aseptic meningitis in Poland, 2011-2014. Pol J Microbiol 2016; 65: 231-5. https://doi.org/10.5604/17331331.1204485.

[45] Ortner B, Huang C, Schmid D, Mutz I, Allerberger F, Yang J, et al. Epidemiology of enterovirus types causing neurological disease in Austria 1999-2007: detection of clusters of echovirus 30 and enterovirus 71 and analysis of prevalent genotypes. J Med Virol 2009; 81: 317-24. https://doi.org/10.1002/jmv.21374.

[46] Jaramillo-Gutierrez G, Benschop K, Claas E, de Jong A, van Loon A, Pas S, et al. September through October 2010 multi-centre study in the Netherlands examining laboratory ability to detect enterovirus 68, an emerging respiratory pathogen. J Virol Methods 2013; 190: 53-62. https://doi.org/10.1016/j.jviromet.2013.02.010. 